\title{
Efficacy of Subconjunctival Injection of Bevacizumab in Regressing Corneal Neovascularisation
}

\author{
Syed Shujaat Ali Shah
}

\begin{abstract}
Objective: To determine the efficacy of subconjunctival injection of bevacizumab in regressing corneal neovascularisation. Study Design: Case series.

Place and Duration of Study: Department of Ophthalmology, Chandka Medical College and Hospital, SMBBMU, Larkana, Pakistan, from October 2015 to March 2016.

Methodology: A total of 95 patients with corneal neovascularisation of more than 3 months' duration and with no history of previous or current anti-VEGF treatment were included in the study. Patients with any other active ocular disease or using any medication were excluded from the study. Subconjunctival injection of bevacizumab $0.05 \mathrm{ml}(1.25 \mathrm{mg})$ was given near the limbus where maximum neovascularisation was seen. The final outcome was assessed after one month of injection; Pre- and post-treatment slit-lamp examination was done and corneal photograph was taken with anterior segment digital camera to see any regression in corneal neovessels from the baseline.

Results: Out of the total 95 patients, $62(65.3 \%)$ were males and $33(34.7 \%)$ were females, $18(18.9 \%)$ patients were known cases of diabetes mellitus and $8(8.4 \%)$ had hypertension. Mean age was $38.46 \pm 10.01$ years. Mean duration of corneal neovascularisation was $1.81 \pm 3.68$ years. After subconjunctival injection of bevacizumab, $14.7 \%$ patients showed regression in corneal neovascularisation.

Conclusion: Subconjunctival injection of bevacizumab is a promising modality for treatment of corneal neovascularisation and may be used in adjunct to other therapies.
\end{abstract}

Key Words: Cornea, Injection, Vascular endothelial growth factor (VEGF), Bevacizumab.

\section{INTRODUCTION}

Cornea contributes the majority of the refractive power of the human eye and also provides a clear entrance pathway to the light rays. Corneal transparency is dependent on many factors like the basal metabolic homeostasis, corneal ultra-structure and its arrangement, tight junctions between corneal epithelial cells and corneal endothelial cells, the corneal endothelial pump and the corneal avascularity. A vessel-free cornea is not only essential for its transparency but also for maintaining its immune privilege, which makes it the best tissue in the human body for grafting. Normally, there are low levels of angiogenic factors and high levels of anti-angiogenic factors in the cornea and any imbalance may lead to neovascularisation. ${ }^{1}$ This imbalance may be caused by infections, inflammations, ischemia, physical trauma, chemical injury and degeneration. Neovascularisation of cornea causes marked impairment of vision and is the main risk factor for rejection after keratoplasty. Approximately, 4\% people in the United States are suffering from corneal neovas-

Department of Ophthalmology, Chandka Medical College and Hospital, SMBBMU, Larkana, Pakistan

Correspondence: Dr. Syed Shujaat Ali Shah, Department of Ophthalmology, Chandka Medical College and Hospital,

SMBBMU, Larkana, Pakistan

E-mail: sayedshujaatali_12@yahoo.com

Received: August 20, 2018; Accepted: November 19, 2018 cularisation (KNV) and another 1.4 million may develop it yearly. ${ }^{2}$ Millions more lose their sight because of corneal neovascularisation worldwide. ${ }^{3}$ Prevalence or incidence of corneal neovascularisation in Pakistan has not been reported yet; but the burden of the problem is assumed to be huge as corneal blindness is the second most common cause of blindness in Pakistan. ${ }^{4}$

VEGF is one of the most important mediators of angiogenesis and is upregulated during neovascularisation. ${ }^{5}$ Keeping this fact in mind, the anti-VEGF (anti-vascular endothelial growth factor) agents have effectively been used to treat many ocular diseases involving neovascularisation, such as, retinopathy of prematurity (ROP), neovascular age-related macular degeneration (nARMD), neovascular glaucoma (NVG), diabetic retinopathy, ${ }^{6}$ and macular edema. ${ }^{7}$ Recently, anti-VEGF agents have been tried for treating corneal neovascularisation with propitious results. Bevacizumab is a very effective recombinant humanised monoclonal antiVEGF antibody. It has been used effectively to treat corneal neovascularisation in many animal models; 8 and furthermore, it has safely been used as an off label drug in human studies as well. Bevacizumab has shown to be of valuable help in cases of high risk corneal transplants by causing regression of the neovessels and improving graft survival. 9

The aim of this study was to determine the efficacy of subconjunctival bevacizumab in regressing corneal neovascularisation. 


\section{METHODOLOGY}

This study was carried out at the Department of Ophthalmology, Chandka Medical College and Hospital, SMBBMU, Larkana, Pakistan, from October 2015 to March 2016. Patients aged $18-60$ years, having corneal neovascularisation of more than 3 months' duration were included in the study after taking written informed consent. Patients having any other active ocular disease, like uveitis, conjunctivitis etc., and those with history of taking previous anti-VEGF treatment or any concurrent local therapy, were excluded from the study.

Slit lamp examination was performed, and corneal photograph was taken with the anterior segment digital camera for assessing corneal neovascularisation and for post-treatment comparison. The eye with corneal neovascularisation was anesthetised with proparacaine (1\%) eye drops, and subconjunctival injection of bevacizumab $0.05 \mathrm{ml}(1.25 \mathrm{mg})$ was given near the limbus where maximum neovascularisation was seen. All the procedures were performed under complete aseptic condition. Patient was then called for follow-up at two weeks and one month intervals after the injection was given. The final outcome was assessed after one month of injection, slit lamp examination was done, posttreatment corneal photograph was taken, and compared with the earlier one. Efficacy was considered as present if minimum $1 \%$ regression in corneal neovessels from the baseline was observed in the corneal photograph. All the collected information were entered into the predesigned proforma. SPSS version 20 was used for data entry and analysis. Frequencies and percentages were calculated for gender, efficacy (Yes/No), diabetes mellitus and hypertension. Mean \pm standard deviation were calculated for age and duration of corneal neovascularisation. Stratification was done for gender, age, diabetes mellitus, hypertension and duration of corneal neovascularisation to control the effect modifiers. The post-stratification Chi-square test was applied taking the $p$-value $\leq 0.05$ as statistically significant.

\section{RESULTS}

Ninety-five eyes of 95 patients with corneal neovascularisation of more than 3 months' duration were included in the study. Out of the total 95 patients, 62 $(65.26 \%)$ were males and $33(34.74 \%)$ were females. Mean age of the patients was $38.46 \pm 10.01$ years, ranging from 18 to 60 years. Mean duration of corneal neovascularisation was $1.81 \pm 3.68$ years ranging from three months to 25 years. Out of the total 95 patients, 18 $(18.95 \%)$ patients were known cases of diabetes mellitus and eight ( $8.42 \%)$ patients were known cases of hypertension.

After subconjunctival injection of bevacizumab into 14 $(14.74 \%)$ eyes, out of the 95 eyes of 95 patients, it showed regression in corneal neovascularisation and were labelled as Efficacy / yes. Stratification of gender showed regression in corneal neovascularization in 9 $(14.52 \%)$ eyes of males and $5(15.15 \%)$ eyes of females, $(p=0.934)$, which was found to be statistically non-significant.

In stratification of age groups, regression in corneal neovascularisation was found in $9(25.71 \%)$ eyes in the age group of $18-35$ years and in $5(8.33 \%)$ eyes in the age group of $36-60$ years, which was statistically significant $(p=0.021)$. Stratification of diabetes and hypertension showed both to be statistically nonsignificant with $p$-value of 0.222 and 0.392 , respectively. When stratification of duration of corneal neovascularisation was performed, regression in corneal neovascularisation was found in $14(15.56 \%)$ eyes with duration 3 months- 5 years and none $(0 \%)$ with duration more than 5 years showed regression in corneal neovascularisation $(p=0.340)$, which was found to be statistically non-significant (Table I).

\begin{tabular}{|c|c|c|c|}
\hline \multirow[t]{2}{*}{ Variables } & \multicolumn{2}{|c|}{ Efficacy of bevacizumab } & \multirow[t]{2}{*}{$p$-value } \\
\hline & Yes $(n)$ & No $(n)$ & \\
\hline \multicolumn{4}{|l|}{ Gender } \\
\hline Male & $9.47 \%(09)$ & $55.79 \%(53)$ & 0.934 \\
\hline Female & $5.26 \%(05)$ & $29.47 \%(28)$ & \\
\hline \multicolumn{4}{|l|}{ Age groups } \\
\hline $18-35$ years & $9.47 \%(09)$ & $27.37 \%(26)$ & 0.021 \\
\hline $36-60$ years & $5.26 \%(05)$ & $57.89 \%(55)$ & \\
\hline \multicolumn{4}{|l|}{ Diabetes mellitus } \\
\hline Yes & $1.05 \% \quad(01)$ & $17.89 \%(17)$ & 0.222 \\
\hline No & $13.68 \%(13)$ & $67.37 \%(64)$ & \\
\hline \multicolumn{4}{|l|}{ Hypertension } \\
\hline Yes & $2.10 \% \quad(02)$ & $6.32 \% \quad(06)$ & 0.392 \\
\hline No & $12.63 \%(12)$ & $78.95 \%(75)$ & \\
\hline \multicolumn{4}{|l|}{ Duration of corneal neovascularisation } \\
\hline 3 months- 5 years & $14.74 \%(14)$ & $80.0 \%(76)$ & 0.340 \\
\hline$>5$ years & $00 \% \quad(00)$ & $5.26 \%(05)$ & \\
\hline Total $(n=95)$ & $14.74 \%(14)$ & $85.26 \%(81)$ & \\
\hline
\end{tabular}

\section{DISCUSSION}

Corneal neovascularisation compromises the corneal transparency leading to the loss of visual acuity and also causes increased susceptibility to graft rejection. Treatment strategies for corneal neovascularisation, presently under use, include both medical and nonmedical therapies including surgical interventions. Medical therapies include Anti-VEGF agents, steroids, nonsteroidal anti-inflammatory agents, tetracycline and immunosuppressive agents like cyclosporine. Nonmedical therapies include, fine-needle diathermy, ${ }^{10}$ laser photocoagulation, ${ }^{11}$ photodynamic therapy, ${ }^{12}$ and surgical procedures like conjunctival autograft, limbal autograft, limbal stem cell transplantation and amniotic membrane transplantation. It is now a proven fact that the vascular endothelial growth factor promotes angiogenesis in cornea. From the time when the first study reported successful regression in corneal neovascularisation in 


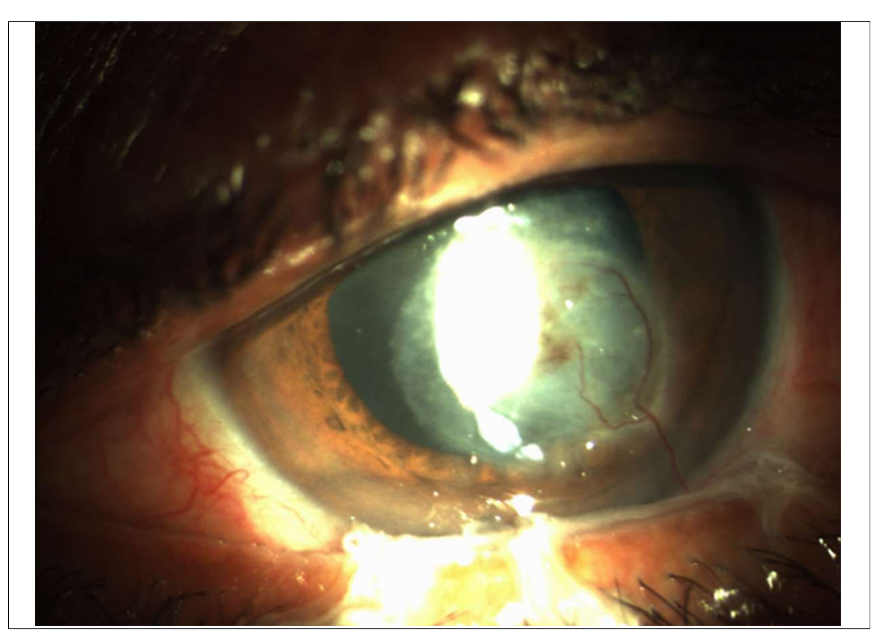

Figure 1: Corneal neovascularisation before subconjunctival injection of bevacizumab.

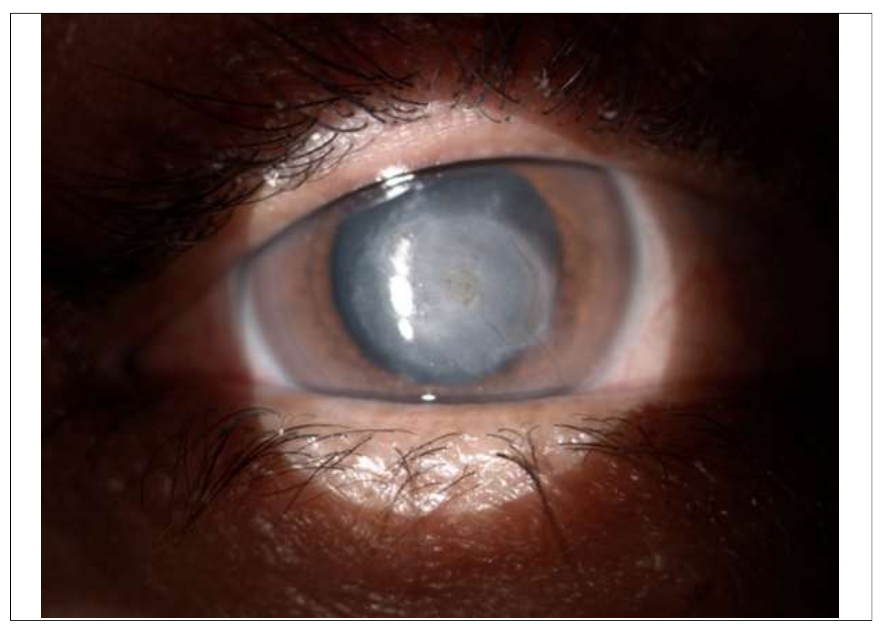

Figure 2: Regression of corneal neovascularisation after subconjunctival injection of bevacizumab.

humans, by using topical anti-VEGF antibodies, ${ }^{13}$ there has been no looking back since then and various antiVEGF agents are now used for the treatment of corneal neovascularisation. Previously, it was thought that topically applied drugs are the safer and more effective mode of therapeutic agent delivery in the treatment of various ocular surface disorders. But, it is now proven that agents given as subconjunctival injection have greater ability to penetrate the intact corneal epithelium than those drugs which are topically applied; 14 and hence, are a better way of dealing with corneal diseases, especially corneal neovascularisation. In this study, bevacizumab which is a humanised monoclonal antibody against all isoforms of VEGF, was used in a single subconjunctival injection for treating corneal neovascularisation.

In this study, males (65.26\%) were in majority in comparison to females (34.74\%). Similar findings, showing male predominance at $60.86 \%$, were also reported by another study from Pakistan, which was conducted on patients with corneal disease. ${ }^{15}$ The male predominance in this study could probably be explained by the fact that the male population of Pakistan is greatly vulnerable not only to outdoor risk factors for developing ocular surface diseases, but also to ocular trauma and professional hazards as reported by Shah et al. ${ }^{16}$

This study also highlights the fact that the patients who are in their middle ages are more susceptible to develop corneal neovascularisation as is clearly evident from the fact that the mean age \pm standard deviation of the patients in this study was $38.46 \pm 10.01$ years, also reported in their study by Lodhi et al. ${ }^{17}$

The final outcome of this study demonstrated that after subconjunctival injection of bevacizumab, eyes of $14.74 \%$ patients showed regression in corneal neovascularisation (Figures 1 and 2). These results are similar to that reported by the study conducted by Mirabelli et al. ${ }^{18}$ Many other studies carried out around the world have reported variable results, although majority of them have reported subconjunctival injection of bevacizumab to be effective in regressing corneal neovascularisation.

Studies conducted on this topic in Pakistan also have reported variable results with Hussain et al. reporting that subconjunctival injection of bevacizumab is effective in regressing the corneal neovascularisation partially, 19 which somewhat resemble the results of this study. On the other hand, Bhatti et al. reported that subconjunctival injection of bevacizumab has not proved to be effective in regressing corneal neovascularisation. 20

Most probably, the overall variation in the results reported in the literature is due to the difference in dosage of the drug used, number of injections given, and the duration of treatment and follow-up. You et al. observed in their study that the increase in dosage of bevacizumab, leads to increase in the efficacy; and hence, better results. 21 They used a double dosage regimen of $2.5 \mathrm{mg}$ and $5 \mathrm{mg}$, on two different treatment groups and noticed more regression of corneal neovessels with the higher dose, ${ }^{21}$ while a dose of 1.25 $\mathrm{mg} / 0.05 \mathrm{ml}$ has been used in this study. In addition, this study has evaluated the patients for the final treatment outcome after one month of a single subconjunctival injection of bevacizumab, in contrast many other studies have used multiple injections, have reported longer duration of treatment and follow-up, ranging from three months to six months. ${ }^{22}$ Based on the above discussion, it may be presumed that the administration of a single injection of bevacizumab, smaller dosage and a shorter duration of treatment and follow-up may have led to the inferior results in this study.

Recent results have indicated that early treatment with subconjunctival injection of bevacizumab had a better therapeutic outcome than delayed treatment, as the corneal neovascularization reduced considerably more in the group treated early. ${ }^{23}$ This finding was also evident in this study as shown by the stratification of age groups 
and duration of corneal neovascularisation. This study also demonstrated through stratification that the presence of diabetes mellitus or hypertension did not affect the results and neither did the gender.

Although a comprehensive comparative study between the newer Anti-VEGF agents would have been ideal in this scenario, but this study had financial limitations, as the newer anti-VEGF agents are expensive, and the limitations of availability as well. A comparative study, conducted on an animal model of corneal neovascularisation was carried out to find the most potent among the anti-VEGF agents in regressing corneal neovascularisation; all delivered as a subconjunctival injection. The study reported bevacizumab to be the most effective agent in regressing corneal neovascularisation. ${ }^{24}$ As it is now a well-known fact that besides VEGF, many other molecules and factors also contribute to the pathogenesis of corneal neovascularisation, which include fibroblast growth factor, angiostatin, endostatin, matrix metalloproteases, platelet derived growth factor, pigment endothelium derived factor, tumor necrosis factor, thrombospondin, insulinlike growth factor, and placental growth factor to name a few. A study conducted in this regard has revealed that combination therapy with bevacizumab and etanercept (a tumor necrosis factor- $\alpha$ inhibitor) has proved to be more potent in regressing corneal neovascularisation than mono-therapy. ${ }^{25}$

Further research is underway to target more of these factors which control other pathways in the pathogenesis of corneal neovascularisation, in addition to the VEGF pathway. Although, uptill now a clear consensus about a perfect treatment of corneal neovascularisation has not been reached, but the future seems to be bright as combination therapies have promised better therapeutic outcomes.

\section{CONCLUSION}

Subconjunctival injection of bevacizumab is an effective treatment option to regress corneal neovascularisation. Additionally, it has also proved to be a rather safe treatment modality. Moreover, using multiple injections of bevacizumab, increasing the drug dosage and duration of treatment, may lead to much better therapeutic outcomes. Recent treatment options for corneal neovascularisation including combination therapies have vowed a future to look forward to with optimistic results.

Disclosure: It is a dissertation based article.

\section{REFERENCES}

1. Hos D, Bukowiecki A, Horstmann J, Bock F, Bucher F, Heindl LM, et al. Transient ingrowth of lymphatic vessels into the physiologically avascular cornea regulates corneal edema and transparency. Sci Rep 2017; 7:7227.
2. Shakiba Y, Mansouri K, Arshadi D, Rezaei N. Corneal neovascularization: molecular events and therapeutic options. Recent Pat Inflamm Allergy Drug Discov 2009; 3:221-31.

3. Dastjerdi MH, Al-Arfaj KM, Nallasamy N, Hamrah P, Jurkunas UV, Pineda R, et al. Topical bevacizumab in the treatment of corneal neovascularization: Results of a prospective, open-label, noncomparative study. Arch Ophthalmol 2009; 127:381-9.

4. Dineen B, Bourne RR, Jadoon Z, Shah SP, Khan MA, Foster A, et al. Causes of blindness and visual impairment in Pakistan. The Pakistan national blindness and visual impairment survey. Br J Ophthalmol 2007; 91:1005-10.

5. Chang JH, Garg NK, Lunde E, Han KY, Jain S, Azar DT. Corneal neovascularization: An anti-VEGF therapy review. Surv Ophthalmol 2012; 57:415-29.

6. Sato T, Morita S, Bando H, Sato S, Ikeda T, Emi K. Early vitreous hemorrhage after vitrectomy with preoperative intravitreal bevacizumab for proliferative diabetic retinopathy. Middle East Afr J Ophthalmol 2013; 20:51-5.

7. Hirose M, Matsumiya W, Honda S, Nakamura M. Efficacy and visual prognostic factors of intravitreal bevacizumab as needed for macular edema secondary to central retinal vein occlusion. Clin Ophthalmol 2014; 8:2301-5.

8. Ozbek Z, Aydin R, Selver OB, Selver MA, Saatci AO, Durak I. Can subconjunctival bevacizumab injection regress corneal neovascularization? Pak J Ophthalmol 2013; 29:21-5.

9. Bhatti N, Qidwai U, Hussain M, Kazi A. Efficacy of topical bevacizumab in high-risk corneal transplant survival. Pak $J$ Med Sci 2013; 29:519-22.

10. Romano V, Steger B, Brunner M, Ahmad S, Willoughby CE, Kaye SB. Method for angiographically guided fine-needle diathermy in the treatment of corneal neovascularization. Cornea 2016; 35:1029-32.

11. Bhatti N, Zaman Y, Rahman Y, Mahar PS, Kamal F, Mazhar-ulHassan, et al. Regression of corneal vascularization by laser treatment. Pak J Ophthalmol 2010; 26:23-7.

12. Kim RY, Chung SK, Kim MS, Ra H. Effects of combined photodynamic therapy and topical bevacizumab treatment on corneal neovascularization in rabbits. Cornea 2016; 35:161520.

13. De Stafeno JJ, Kim T. Topical bevacizumab therapy for corneal neovascularization. Arch Ophthalmol 2007; 125:834-6.

14. Dastjerdi MH, Sadrai Z, Saban DR, Zhang Q, Dana R. Corneal penetration of topical and subconjunctival bevacizumab. Invest Ophthalmol Vis Sci 2011; 52:8718-23.

15. Bhatti N, Fawad MU, Hussain M, Qidwai U, Hasan M, Rehman A. Three years clinical audit of patients presenting in cornea clinic at a tertiary care teaching hospital in Karachi. Pak J Ophthalmol 2011; 27:191-4.

16. Shah SIA, Shah SA, Rai P, Abbasi SA, Fatima H, Soomro AA. Etiology of infectious keratitis as seen at a tertiary care center in Larkana, Pakistan. Pak J Ophthalmol 2016; 32:48-52.

17. Lodhi AA, Murtaza, Ahmed M, Ahmed N, Haider G, Junejo SA, et al. Role of tetracycline in corneal neovascularization. Pak J Ophthalmol 2015; 31:150-3.

18. Mirabelli P, Peebo BB, Zeroudaki M, Koulikovska M, Lagali N. Early effects of dexamethasone and anti-VEGF therapy in an inflammatory corneal neovascularization model. Exp Eye Res 2014; 125:118-27. 
19. Hussain I, Aftab AM. Role of sub-conjunctival bevacizumab in regression of corneal neovascularization. Pak J Ophthalmol 2014; 30:99-102.

20. Bhatti N, Qidwai U, Fawad U, Hussain M, Rehman A, Mazhar UI Hasan. Side effects and effectiveness of subconjunctival bevacizumab injection in patients with corneal neovascularization. Pak J Ophthalmol 2012; 28:33-7.

21. You IC, Kang IS, Lee SH, Yoon KC. Therapeutic effect of subconjunctival injection of bevacizumab in the treatment of corneal neovascularization. Acta Ophthalmol 2009; 87:653-8.

22. Zaki AA, Farid SF. Subconjunctival bevacizumab for corneal neovascularization. Acta Ophthalmol 2010; 88:868-71.
23. Petsoglou C, Balaggan KS, Dart JKG, Bunce C, Xing W, Ali RR, et al. Subconjunctival bevacizumab induces regression of corneal neovascularisation: A pilot randomised placebo-controlled double-masked trial. Br J Ophthalmol 2013; 97:28-32.

24. Sener E, Yuksel N, Yildiz DK, Yilmaz B, Ozdemir O, Caglar Y, et al. The impact of subconjuctivally injected EGF and VEGF inhibitors on experimental corneal neovascularization in rat model. Curr Eye Res 2011; 36:1005-13.

25. Ozdemir O, Altintas O, Altintas L, Yildiz DK, Sener E, Caglar Y. Effects of subconjunctivally injected bevacizumab, etanercept, and the combination of both drugs on experimental corneal neovascularization. Can J Ophthalmol 2013; 48:115-20.

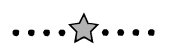

